\title{
The high expression of elastases, observed in apelin deficient mice, could contribute to premature wear of elastic fibers and vascular stiffness.
}

\section{Beatrice Romier}

University of Reims Champagne-Ardenne

\section{Cedric Dray}

University of Toulouse

Amandine Wahart

University of Reims Champagne-Ardenne

Thinhinane Hocine

University of Reims Champagne-Ardenne

\section{Laetitia Vanalderwiert}

University of Reims Champagne-Ardenne

Alizée Dortignac

University of Toulouse

Christian Garbar

Institut Jean Godinot

Corinne Garbar

Institut Jean Godinot

Camille Boulagnon

Centre Hospitalier Universitaire De Reims

Nicole Bouland

University of Reims Champagne-Ardenne

Pascal Maurice

University of Reims Champagne-Ardenne

\section{Amar Bennasroune}

University of Reims Champagne-Ardenne

Herve Sartelet

University of Reims Champagne-Ardenne

Laurent Martiny

University of Reims Champagne-Ardenne

\section{Laurent Duca}

University of Reims Champagne-Ardenne

Philippe Valet 
University of Toulouse

Sebastien Blaise ( $\square$ sebastien.blaise@univ-reims.fr)

University of Reims Champagne-Ardenne

\section{Research Article}

Keywords: Apelin, Elastin, Cathepsin S, Adiposity, Vascular stiffness

Posted Date: February 11th, 2021

DOl: https://doi.org/10.21203/rs.3.rs-153958/v1

License: (1) This work is licensed under a Creative Commons Attribution 4.0 International License. Read Full License 


\section{The high expression of elastases, observed in apelin deficient mice, could contribute to premature wear of elastic fibers and vascular stiffness.}

Beatrice Romier ${ }^{1,6}$, Cédric Dray ${ }^{2,6}$, Amandine Wahart ${ }^{1}$, Thinhinane Hocine ${ }^{1}$, Laetitia Vanalderwiert ${ }^{1}$, Alizée Dortignac ${ }^{2}$, Christian Garbar ${ }^{3}$, Corinne Garbar ${ }^{3}$, Camille Boulagnon ${ }^{1,4}$, Nicole Bouland ${ }^{5}$, Pascal Maurice ${ }^{1}$, Amar Bennasroune ${ }^{1}$, Hervé Sartelet ${ }^{1}$, Laurent Martiny ${ }^{1}$, Laurent Duca ${ }^{1}$, Philippe Valet ${ }^{2,6 *}$, Sébastien Blaise ${ }^{1,6 *}$

${ }^{1}$ UMR CNRS 7369 MEDyC, University of Reims Champagne-Ardenne, Reims, France.

${ }^{2}$ Institut RESTORE, Université de Toulouse, CNRS U-5070, EFS, ENVT, Inserm U1301, Université Paul Sabatier, Toulouse, France.

${ }^{3}$ Departement of Biopathology, Jean Godinot Institut, Regional Cancer Control Center, Reims, France

${ }^{4}$ Laboratory of Biopathology, University Hospital Center, Reims, France.

${ }^{5}$ Laboratory of Anatomy and Pathologies, Faculty of Medicine, University of Reims ChampagneArdenne, Reims, France.

${ }^{6}$ equal contributions

* Corresponding authors: Sébastien BLAISE, UMR CNRS/URCA 7369, UFR Sciences Exactes et Naturelles, Moulin de la Housse, BP 1039, 51687 Reims Cedex 2, Phone: (+33) 326913435 / Fax: (+33) 326918366 / sebastien.blaise@univ-reims.fr Philippe VALET, Institut RESTORE, 4bis ave Hubert Curien, 31100 Toulouse, France. Phone : (+33) 534609502 / philippe.valet@inserm.fr 
Short Title: Apelin deficiency induces elastolysis

Disclosures: The authors disclose no conflicts.

Conflict of Interest: No potential conflict of interest relevant to this article were reported.

Author contributions:

Conceptualization, Funding acquisition, Project administration, Ressources, Supervision, Validation, Vizualization, Writing - original draft, Writing: $S B, B R, P V, C D$

Formal analysis, Investigation, Methodology: $S B, B R, P V, C D, L V, H S, A D, C B, N B, C h G, C o G, A W, T H$ Review \& editing: $S B, B R, P V, C D, P M, A B, L M, L D$

\section{ACKNOWLEDGMENTS}

We wish to thank Marie-Line Sowa, Fanja Rabenoelina and Olivier Bocquet for technical help. This work was supported by funding from the CNRS, the University of Reims Champagne Ardenne, the Région Grand-Est (OMAGE program, France), European funds (FEDER). AW was supported by research grants from the Région Champagne Ardenne. TH was support by research grants from URCA. We would like also to acknowledge for their expertise the Anexplo platform (phenotypage) of the UMS006 Toulouse and the staff of animal facilities. 


\section{ABSTRACT}

Vascular stiffness is often the main cause of arterial hypertension and its complications including atherosclerosis, observed during obesity. However, the mechanisms leading to this rigidity or the preventing factors are misknown. We hypothesized that apelin, known for its beneficial effects on lipid, inflammatory, and vascular metabolism, could be a protective factor against vascular stiffness. We used mice deficient for the apelin gene (KO-APL) and compared with wild-type mice (WT) at the level of metabolic markers and inflammations of white adipose tissue (WAT), as well as aortic functional and anatomical parameters.

KO-APL mice developed an inflammation associated with significant remodeling of WAT, in particular with the protease expressions such as neutrophil elastase or cathepsin S. From a vascular point of view, these same elastases are involved in the fragmentation of elastic fibers, explaining the increase in vascular velocity of pulse wave and arterial hypertension. Interestingly, univariate correlation analysis showed that the inflammation markers and protease expression of WAT were associated with remodeling of the vascular wall.

Our results suggest that the modifications induced by the absence of apelin particularly in WAT, could facilitate the expression of elastases and the rupture of elastic fibers, necessary to maintain elastance. This discovery is fundamental because the synthesis of elastic fibers stops as of adolescence and is not renewed during the entire life of human. The preservation of these fibers is therefore critical in maintaining vascular homeostasis. Thus, Apelin could be an interesting therapeutic route to protect the premature wear of elastic fibers.

Keywords: Apelin, Elastin, Cathepsin S, Adiposity, Vascular stiffness 


\section{INTRODUCTION}

A veritable global scourge, the metabolic syndrome, also known as $\mathrm{X}$ syndrome, denotes the coexistence of several health disorders of lipid (fatty liver), carbohydrate (insulin resistance) or vascular origin associated with excess weight (obesity), in the same individual. Health authorities estimate that $20 \%$ of the general population has at least 3 metabolic disorders among obesity, Non-Alcoholic SteatoHepatitis (NASH), dyslipidemia, insulin resistance or high blood pressure[1].All of these metabolic disorders considerably increase the risk of type 2 diabetes and cardiovascular complications including atherosclerosis, abdominal aortic aneurysm (AAA) and strokes. There is currently no drug treatment that can "cure" exclusively the metabolic syndrome. However, it is possible to specifically treat blood hypertension, cholesterol excess or even type 2 diabetes to limit the risk of cardiovascular disease. The lack of effective therapy resists in a still partial understanding of the mechanisms connecting the various pathologies. An international consensus is emerging on the fact that adipose tissue would be the main key of the solution. [2]. Indeed, the accumulation of fat in visceral adipose tissue is a predictor of metabolic but also inflammatory changes. Considered as an energy reserve tissue, white adipose tissue has been recognized for twenty years as an endocrine organ which plays a major role in the physiology of immunity and the pathophysiology of inflammation, by secreting hormones grouped under the term of adipokines such as leptin, adiponectin or apelin. Altered expression or secretion of these adipokines can be a vector of a low-level chronic inflammatory state which could play a central role both in the cardiovascular complications of obesity and in insulin resistance, a factor risk of type 2 diabetes. Among adipokines, apelin play a major role in chronic metabolic [3] as well as cardiovascular disorders. Indeed, this ligand for APJ receptor, a G proteincoupled receptor, could regulate its downstream signaling and plays an important part in the occurrence and development of obesity, type 2 diabetes [4] , hypertension, and other cardiovascular diseases such as atherosclerosis [5]or AAA [6]. Nevertheless, the mechanism supposes a direct effect of apelin and APJ on cells of blood vessel. Thus, several study showed that the apelin/APJ system could decrease blood pressure through nitric oxide (NO)-dependent pathway [7]. Moreover, apelin could increase ACE2 promoter activity. This overexpression of ACE2 expression could accelerate the hydrolysis of angiotensin II (Ang-II), a strong vasoconstrictor. However, apelin is also a major regulator of inflammatory factors [8]. Therefore, it is conceivable that apelin could have another role via inflammatory modulation. Our hypothesis is that the inflammation generated by the absence of apelin, in particular in the adipose tissue could contribute to the recruitment of proteases. The proteases such as the neutrophil elastase, cathepsin S, MMP2, MMP9 ... are described as factors responsible for the fragmentation of elastic fibers [9]. Unlike many proteins, elastic fibers are complex multi-protein structures whose synthesis stops in adolescence. At this stage, any individual then presents a stock of 
defined fibers which will be subjected to mechanical and enzymatic stresses and resulting in more or less premature wear [10]. Thus, premature aging of these elastic fibers is responsible, among other things, for vascular stiffness, in particular of the aorta and is believed to be the cause of the vascular complications observed in obese patients [11]. Those diseases are major complications and the main causes of death observed in obese and / or diabetic subjects. To answer our hypothesis, we used mice lacking apelin and characterized their white adipose tissue as well as their aortic wall.

\section{RESULTS}

Apelin deficiency increases inflammatory status of white adipose tissue: While the weight (Figure 1A) and the lean mass (Figure 1B) measured from EchoMRI's approach, remain unchanged, we demonstrate that KO mice develop their fat mass (Figure $1 \mathrm{C}$ ) associated with an overexpression of adipokines mRNA such as leptin (Figure 1D). Hematoxylin -Eosin staining shows that the sizes of lipid droplets are increased (Figure 1E). This increase is also associated to increase of inflammatory markers such as macrophage (MCP1 or F4/80) and cytokines such as TNF $\alpha$, IL $\beta$. TGF $\beta$ is not affected by the absence of apelin expression (Figure 1F). In several tissues, inflammation is known to favor the expression of proteases. Thus, in Apelin KO mice, the expressions of cathepsin S, neutrophil elastase or MMP9 are significantly increased (Figure 1G). In parallel, we observed that expression of their inhibitors, cystatin C, serpin and TIMP-1 respectively, are also influenced by apelin (Figure $1 \mathrm{H}$ ). Nevertheless, the ratios between the protease expression and its natural inhibitor show that the balance tilts towards protease activity (Figure 11). These results confirm that apelin defect is responsible of lipogenic metabolism and inflammatory and protease expression phenotypes. Those data suggest that apelin expression favors an activation of lipogenic metabolism which may be responsible for vascular complications.

Apelin deficiency impaired aorta rigidity: Apelin has been described as an hormone involved in the onset of atherosclerosis and abdominal aortic aneurysms (AAA). However, these cardiovascular pathologies are regularly the consequence of vascular rigidity and / or high blood pressure. Furthermore, one of the first cardiovascular complications seen in patients with diabetes and / or obesity is high blood pressure. Thus, we evaluated the arterial pressures on vigil animals with the method of "tail cuff blood pressure" carried out every day during 4 days (at the same period of the day). The diastolic pressures are similar between the group KO and WT with a value around $55 \mathrm{mmHg}$ (Figure 2A, 2B). In contrast, the systolic (Figure 2A, 2B) and mean arterial (MAP, Figure 2C- 2E) levels were significantly elevated during the 4 days of measurements in the KO mice compared to the WT mice. These results show that the absence of apelin promotes arterial hypertension. In addition, we 
noted an increase in pulsed pressure (Figure $2 \mathrm{E}, 2 \mathrm{~F}$ ) in these mice which could mean an increase in vascular rigidity. While heart frequency (Figure $2 \mathrm{G}$ ) is not modified, the measurement of the pulse wave velocity (Figure $2 \mathrm{H}$ ) carried out at the level of the descending thoracic aorta, increased in these same mice, we can affirm that the arterial hypertension can be explained by an increased rigidity of the descending aortic wall. These results suggest that the absence of apelin causes rigidity of aorta wall which may be related to the inflammation and/or protease expressions induced in the fatty tissue. Thus, Z-test shows that systolic, pulsed or pulse wave pressures can be correlated to the expression of inflammatory markers observed in adipose tissue such as MCP1 (Figure $2 \mathrm{I}$ to $2 \mathrm{~K}$ ) or elastases such as cathepsin S (Figure $2 \mathrm{~L}$ to $2 \mathrm{~N}$ ) or neutrophil elastase (Figure $2 \mathrm{O}$ to $2 \mathrm{Q}$ ).

Apelin deficiency impaired collagen expression in aorta wall: Vascular stiffness can be explained by a remodeling of the vascular wall and in particular of the ECM. From protein constitutive of scaffold of extracellular matrix blood vessels, collagen molecules are one major components, involved in strength and resistance to stretching. These properties depend on the composition, as well as the diameter of the collagen fiber. We analyzed vascular collagen by measuring total collagen in aorta of mice expressing or not apelin. While collagen I mRNA is not overexpressed (figure $3 \mathrm{~A}$ ), an accumulation of collagen protein is observed by collagen assay (Figure 3B) or by picrosirius staining (Figure $3 \mathrm{C}$ ) in $\mathrm{KO}$ APL mice compared to the control condition. This collagen accumulation in adventice layer (Figure $3 \mathrm{C}$ and Figure 3E) may be due to an over-expression of collagen III. Indeed, observations of collagen under polarized light (Figure 3D) and semi-quantification (Figure 3F), show collagen accumulation corresponds mainly to collagen fibers of type III rather than type I. Interestingly, we performed a correlation study (univariate $\mathrm{Z}$ test) between the data concerning the characterization of collagen by histological approaches and molecular markers observed in adipose tissue. Therefore, we observed a good correlation between collagen expression and inflammation marker (MCP1, Figure 3G) and a high correlation with protease expressions (in particularly with neutrophil elastase (72.8\%, Figure $3 \mathrm{H}$ ), cathepsin S (82.9\%, Figure 3I)or MMP9 (71.7\%, Figure 3J)). Those correlations suggest in absence of apelin, a real association between ECM remodeling of adipose and vascular tissues. In parallel, we performed a similar univariate correlative study (Z-test) to determine a potential association between the evolution of aortic collagen, and functional parameters observed in WT and APL-KO groups. Thus, the supplementary data 1 shows that the determination of the total collagen (suppl data $1 \mathrm{~A}$ ) or the quantification by the picrosirius (suppl data 1B) seem very few correlated with the systolic and pulsed pressures or the pulse wave velocity. These different correlations suggest that the aortic stiffness demonstrated in Figure 2 could be generated by other proteins forming vascular ECM according to Duca et al[12]. 
Apelin deficiency impaired elastic lamella in aorta wall: In the arterial system, the ECM becomes more rigid with physiological or pathological aging, due to significant changes in its composition, such as the decrease of elasticity of lamellae[13]. With an histological approach (hematoxylin-eosin staining), on transverse sections of descending thoracic aorta (Figure 4A), we noted a slight increase in thickness of the tunica adventitia (which may be explained by the accumulation of collagen, Figure 2) and the tunica media in mice lacking apelin (Figure 4B), similar to what is found in elderly animals. The autofluorescence study of elastin (Figure 4C) shows no significant difference in the number and thickness of elastic fibers (Figure 4D). However, there are many ruptures of the elastic lamellas constituting the media (Figure 4C). The rupture quantification was carried out by dividing the vessel into 4 parts (quadrants) as described by Trachet et al[14]. We were thus able to count these ruptures by several approaches. This count by quadrant shows an increase in the number of fractures per lamella in apelin deficient condition (Figure 4D). This count is inversely correlated with the decrease in autofluorescence of elastin observed in KO mice (Figure 4E). Interestingly, we noted that all the elastic lamellae are affected by the ruptures (Figure 4F), however the lamellae at the ends of the tunica media (lamella 1 and 6) seem to be the least impacted. Finally, we measured the production of elastin derived peptides (EDP), resulting from the fragmentation of the elastic lamellae and found in the plasma (Figure 4G). Once again, we find more peptides released by these ruptures in individuals with deficient expression of apelin than in control animals. This data is strongly positively correlated with the count of lamellar ruptures and negatively with the autofluorescence of elastin, testifying that the histological and biochemical approaches are sufficient to assess the fragmentation of the elastic lamellae. Numerous studies mention that arterial stiffness is the consequence of an increase in collagen synthesis and a decrease in the elasticity of the media. To confirm that the absence of apelin is at the origin of this rigidity, we carried out a ratio between the quantifications of elastin (figure 4C) obtained by autofluorescence and the quantification of collagen obtained by labeling with picrosirius (figure $3 \mathrm{C}$ ). Because the ratio is in favor of collagen in APL KO mice, Figure $4 \mathrm{H}$ demonstrates a vascular rigidity in our model. Interestingly, we performed a Z-test evaluation between this ratio and NE/serpin or Cathepsin S/Cystatin C determined in white adipose tissue and we observed in figure $4 \mathrm{~J}$, a correlation superior to $85 \%$ between the expression of elastase of white adipose tissue and the vascular rigidity index.

Finally, an evaluation of the expression of the components of the elastic fibers was carried out by qPCR. While the expression of elastin is decreased, those of fibrillin 1 and fibulin 5 are significantly increased (Figure 4I). This expression could mean that the smooth muscle cells of the media or the fibroblasts of the adventitia would allow a neo-synthesis of microfibrils in order to compensate for the loss of elasticity by the number of ruptures of the elastic lamellae. From a functional point of view, we wanted 
to know if hypertension or increased aortic velocity could be attributed to the fragmentation of elastic fibers in KO APL mice. For this, we carried out a univariate correlation study ( $Z$ test) on the $8 \mathrm{KO}$ animals and the 8 WT animals, between the different pressures measured in Figure 2 and the quantification of ruptures by the histological approach (Figure 4D) and by evaluation of EDP production (Figure 4E). The linear regression (suppl data 1C and 1D) shows a significant correlation, greater than $80 \%$ for systolic and pulsed pressures and almost $60 \%$ for pulse wave velocity. This statistical approach therefore demonstrates that the fragmentation of elastic fibers, potentially influenced by white adipose tissue, is responsible for vascular hypertension.

ECM remodeling may be explained by the increase of elastase activity: A quarter of apelin KO animals present high ruptures of lamellae the most inner (near of luminar aorta, Figure 5A). This observation suggests that elastic lamellae fragmentations induced by the apelin expression deficiency, may responsible for severe aneurysms. Therefore, we studied the presence or not of proteases (elastinolytics, collagenolytics ...) which might explain the fragmentation of the elastic lamellae in our model. While the expression of MMP12 is significantly reduced (Figure 5B), proteases such as MMP9, the neutrophil elastase and cathepsin $S$ expressions are drastically increased. This result suggests significant proteolytic activity in the vessel. Therefore, we measured the plasma activity of neutrophil elastase and Cathepsin S (Figure 5C) in our model and found that the activities were significantly increased in the absence of apelin. In addition, these activities are significantly correlated with the fragmentation of the elastic lamellae as well as with the production of EDP (Figure 5D and 5E). The immunostaining performed against cathepsin S (Figure 5F) shows that cathepsin S is essentially present in the junction between the adventitious tunica and tunica media (as shown by the arrowheads) and also in the lumen of the aorta in contact with the intima tunica and could explain elastin fragmentations (Figure $5 \mathrm{H}$ ). This result therefore suggests that cathepsin could come from circulating inflammatory cells (such as macrophages) or MEC remodeling observed by the excess adipose tissue (Figure 1). In addition, we show also a strong increase of TGF $\beta$ expression in media (Figure 5G), correlated to lamellae ruptures on one hand (Figure $5 \mathrm{I}$ ) and on the other hand to cathepsin $\mathrm{S}$ expression at $70 \%(p=0.0018)$ and picrosirius staining at $76.8 \%(p, 0.0063)$.

Finally, we performed a similar univariate correlative study (Z-test) to determine a potential association between the evolution of aortic collagen, and functional parameters observed in WT and APL-KO groups. Thus, the supplementary data 1 shows that the determination of the activity (suppl data $1 E$ ) or the expression of Cathepsin S (suppl data 1F) seem highly correlated with the systolic and pulsed pressures or the pulse wave velocity. These different correlations suggest that in our model, cathepsin $\mathrm{S}$ may be a major determinant of the aortic stiffness demonstrated in Figure 2. 


\section{DISCUSSION}

With 17.7 million deaths per years, cardiovascular diseases (CVD) are the leading cause of decease in the world accounting for $31 \%$ of total global mortality [10, 15]. Among those diseases, atherosclerosis and abdominal aortic aneurysms (AAA) are particularly deadly and only surgical repairs are approved medical therapies and practiced[16, 17]. The other therapeutic approaches have unfortunately not brought obvious improvements.. This is the reason why a better understanding of cell deregulation and of the signaling networks responsible for the formation and progression of CVD is necessary for the discovery of new and effective therapies. It is now clearly established that the metabolic syndrome, including obesity and / or diabetes, is a key element in the development of CVD $[7,18,19]$. Among the adipocyte factors involved the secretion of apelin could play a major protective role. Apelin is an adipokine, involved in the regulation of obesity, insulin resistance and also the inflammatory factors $[20,21]$. In addition, we show in this study that apelin plays a major role in extracellular matrix remodeling of WAT, in particular in the production of proteases and their natural inhibitors. Thus, the absence of apelin expressions is in favor of the synthesis of proteases such as neutrophil elastase or cathepsin $S$ considered as a marker of obesity $[22,23]$ while inhibitors such as serpin is reduced. The increased cystatin $C$, described as correlated with inflammation could be explained as an upward adaptation of cathepsin $\mathrm{S}$ in order to limit its impact on adipose tissue and the surrounding ones. It is now accepted that obesity is strongly associated with the degree of CVD. However, the molecular players associating adipose tissue and the vascular compartment remain to be determined. In our study, we demonstrate a strong association between the level of expression of proteases such as cathepsin $\mathrm{S}$ and neutrophil elastase in adipose tissue and vascular parameters in particular the aorta stiffness. Clinically, the stiffness of the arteries is a predictor of death from stroke or AAA, independently of any other predictor [23]. The cytokines IL6, IL1 $\beta$ or TNF $\alpha$ have been widely described as factors responsible for the progression of those vascular diseases. These inflammatory factors, regulated by the presence of Apelin, induce within vascular tissue or macrophages, the expression and secretion of many proteases including metalloproteases (MMP2, MMP9) or cathepsins.

Under physiological conditions, cathepsins are essentially lysosomal proteases active in an acidic environment. This family includes 11 subtypes and most are cysteine proteases. Unlike the majority of these proteases, cathepsin $\mathrm{S}$ can be activated at neutral $\mathrm{pH}$, thus extending its biological role to the extracellular compartment. In obese patients and animal models, the cathepsin S is expressed mainly in the vascular stroma fraction of adipose tissue, in particular at the level of the macrophages, but also in the adipocytes themselves [24]. The overexpression of cathepsin S in the obese adipose tissue is therefore based both on the increased presence of macrophages and on the increase in adipocyte 
expression. While, the secretion of proinflammatory cytokines (TNF $\alpha$, IL1 $\beta, I L 6, \ldots .$.$) by macrophages$ could stimulate the expression of cathepsin $\mathrm{S}$, the factors responsible involved in adipose tissue during obesity are still poorly understood. The results obtained in this study show for the first time that the apelin expression would be decisive in the rise of proinflammatory factors. At the basal membrane of the blood vessels, secreted cathepsin S cleaves several extracellular matrix proteins, including laminin, collagen, and, preferentially, elastin, which generate bioactive elastin peptides [10]. This remodeling of ECM contributes to atherosclerotic lesions and inflamed valves, and in the disruption of the tissue layer, mesenchymal cells [vascular smooth muscle cells (SMCs) or valvular myofibroblasts] proliferate and calcify.

Elastin is a fibrillar protein found in elastic tissues, such as the large vessels, the lung and the skin. At the level of the aorta, elastin forms with the fibrillar proteins (ie. Fibulin 4/5, fibrillin 1/2, LTBP) the concentric elastic laminae at the level of the media [25]. This set, elastin and fibrillar proteins, confer the resilience of the tissues and thus transforms the intermittent blood flow coming from the heart into a continuous flow in the arterioles or the capillaries. Therefore, the elastin integrity is determinant to regulation of blood pressure [12]. Elastin has remarkable physicochemical properties such as hydrophobicity, thus explaining its elastic power, but also resistance to extreme thermal and chemical conditions. This is why elastin is the most durable element of ECM with a half-life of 70 years. Elastin, like fibrillar proteins, is synthesized until puberty and their neo-synthesis in adults and the elderly is almost zero. Thus, during the whole of his life, the individual lives with a defined stock of elastic lamellae. This means that the physiological alternation between stretching and loosening of the elastic lamellae causes mechanical wear. The chronic inflammation induced by insulin resistance and / or obesity and the release of elastase like cathepsin S could accelerate and increase the ruptures of the lamellae as we demonstrate in this study. In addition, the absence of an effective neosynthesis of elastic fibers generates rigidity but also fragility of the aortic wall, leading to blood hypertension on the one hand and on the other hand to the progression of CVD [12]. Moreover apelin has been described in many models as a negative regulator of fibrosis. The cellular mechanisms show that activation of the APJ receptor leads to a reduction in the fibrotic response induced by TGF- $\beta$. However, recent studies[5, 26-28] have shown that cathepsin S (such as TNF $\alpha$, IL1 $\beta$ ) plays a role in the signaling of TGF- $\beta$, the differentiation of myofibroblasts and the creation of ECM, in particular in the synthesis of collagen. Finally, to make the system more complex, TGF- $\beta$ is also an activator of cathepsin $S$. Consequently, in such apelin-deficient model, the induced inflammation could promote selfamplification of the TGF- $\beta$ - Cathepsin $S$ axis, thus contributing to the development of blood hypertension and/or the aggravation of atherosclerosis and AAA. 
To conclude, we have shown in this study that the absence of apelin plays a major role in the induction of vascular stiffness, the first factor involved in the occurrence of arterial hypertension and its complications. The fragmentation of the elastic fibers by the local or remote induction (ie, WAT) of elastases such as cathepsin $\mathrm{S}$ is believed to be the cause of this vascular stiffness. We propose that the inflammation caused by the absence of apelin could then be decisive for the activation of elastases. It would therefore be appropriate to consider apelin as a possible therapeutic way, which might limit / protect inflammation and therefore the activation of proteases in adipose and / or vascular tissues.

\section{MATERIALS AND METHODS}

Animal models: All animal experiments were performed in compliance with the French Institute of Medical Research (INSERM) guidelines and were approved by the animal ethics committee of Toulouse university on the use and care of animals for animal research. Moreover, the study was carried out in compliance with the ARRIVE guidelines. Twelve-week-old apelin knock-out $(n=8)$ and wild-type mice $(n=8)$ were caged in a 12:12 hour light/dark cycle in a temperature- and humidity-controlled environment. All mice were allowed ad libitum access to normal diet (AIN-93M rodent diet, Special Diet Service, UK) and water during the experimental period.

Plasma assays. According to Blaise et al. [29], evaluations of EDP concentrations were performed using commercially available kits (Biocolor, County Antrim, UK; respectively). NE activity assay and Cathepsin activity assay commercialized by Abcam (Cambridge, U.K.) were used to measure neutrophil elastase and Cathepsin S activities, respectively, according to Romier et al[9].

Blood pressure assay by tail-cuff: Mice systolic blood pressure was monitored by the tail cuff technique with the aid of a computerized system (BP 2000 Blood Pressure Analysis System, Visitech System, Apex, NC). Preconditioning of mice was performed during the week before the final blood pressure measurements. On each day of blood pressure determination, 10 measurements were obtained and averaged for each mouse. All animals were tested by an investigator blinded to the treatment of the animals. We measured blood pressure one time per day for four consecutive days. If the systole measurements of the KO mice were higher than those of the control mice on each of the four days, we considered the mice to be hypertensive.

Velocity assay: Doppler ultrasound (Indus Mouse Doppler System, Webster, TX) was performed under gas anesthesia with $2 \%$ isoflurane (Centravet). Mice were placed supine on a heating board with legs fixed on electrocardiographic electrodes to follow vital constants. Doppler probes were placed on the 
transverse aortic arch $(10 \mathrm{MHz})$ and abdominal aorta $(20 \mathrm{MHz})$ and the distance between the probes was determined with a precision caliper. Pre-ejection time, the time between the R-wave of the electrocardiogram and the foot of the Doppler signal, was determined by using at least 8 signals for each measure. Aortic PWV (aPWV) was calculated by dividing the distance $(\mathrm{cm})$ between the probes by the difference in pre-ejection times (milliseconds) of the thoracic and abdominal regions.

Body fat mass composition. To determine fat and lean mass, mice were placed in a clear plastic holder without anesthesia or sedation and inserted into the EchoMRI-3-in-1 system from Echo medical systems (Houston, TX, USA).

Histology: Aorta samples were embedded in paraffin. Three transversal aorta sections were stained with hematoxylin and eosin (H\&E) or with picrosirius as described previously by Pooya et al.[30] . Picrosirius staining was observed by microscopy under polarized light or not (Leica). Picrosirius staining, thickness of tunica media and adventice were quantified using the ImageJ software from three distinct sections per animal $(n=16)$. Expression of cellular and ECM proteins were assessed by immunolabeling aortic tissue sections $(4 \mu \mathrm{m})$ with primary antibodies targeting Cathepsin S (sc-271619, Santa Cruz). Visualization and image acquisition were achieved through microscopy and analyzed with ImageJ software. Elastin was measured by autofluorescence recorded by excitation at $488 \mathrm{~nm}$ in tissue sections according to Romier et al[9]. Each aorta section was divided in four quadrants as descripted by Trachet et al.[14], favoring the quantifications of lamellar rupture, thickness and number of fibers and autofluorescence of elastin by ImageJ software.

Gene expression analysis: Analysis by qPCR was performed as previously described. Briefly, total RNA were extracted using Trizol reagent (Eurobio Scientific). RNA concentration was measured using a Nanodrop system (ThermoFisher Scientific). The 260/280 ratio calculated from Nanodrop software was also used to evaluate protein contamination. Complementary DNA (cDNA) was generated using Verso cDNA kit (ThermoScientific). Real-time PCR was performed using SYBR Green on a BioRad CFX96 Real Time System. $5 \mu$ l of CDNA $(1 / 10)$ and $0.7 \mu$ l of each forward and reverse primers at $3 \mu \mathrm{M}$ were used for qPCR, with cycling conditions: $95^{\circ} \mathrm{C}$ for $15 \mathrm{~min}$, and 40 cycles of $95^{\circ} \mathrm{C}$ for $10 \mathrm{sec}, 60^{\circ} \mathrm{C}$ for 60 sec. RNA expression was normalized to the housekeeping gene 36B4 and RPS26 and relative gene expression was calculated using $2-\Delta \Delta C T$ method. Sequences for qPCR primers are listed in Table 1.

Statistical analyses: Statistical analyses were conducted using Statview Software. Comparisons between groups ( $8 \mathrm{KO}$ mice and $8 \mathrm{WT}$ mice) are presented as mean \pm standard error of the mean (SEM). Univariate correlation was performed by a Z-test regressions. Comparisons among normal 
distributions were made by $\mathrm{t}$-test, and by Mann-Whitney $\mathrm{U}$ test among non-normal distributions. $\mathrm{P}<0.05$ was considered to indicate significance.

\section{REFERENCES}

1. Singleton, J.R., et al., Microvascular complications of impaired glucose tolerance. Diabetes, 2003. 52(12): p. 2867-73.

2. Man, A.W.C., et al., Perivascular Adipose Tissue as a Target for Antioxidant Therapy for Cardiovascular Complications. Antioxidants (Basel), 2020. 9(7).

3. Bertrand, C., P. Valet, and I. Castan-Laurell, Apelin and energy metabolism. Front Physiol, 2015. 6: p. 115.

4. Castan-Laurell, I., B. Masri, and P. Valet, The apelin/APJ system as a therapeutic target in metabolic diseases. Expert Opin Ther Targets, 2019. 23(3): p. 215-225.

5. Chun, H.J., et al., Apelin signaling antagonizes Ang II effects in mouse models of atherosclerosis. J Clin Invest, 2008. 118(10): p. 3343-54.

6. Wang, W., et al., Apelin protects against abdominal aortic aneurysm and the therapeutic role of neutral endopeptidase resistant apelin analogs. Proc Natl Acad Sci U S A, 2019. 116(26): p. 13006-13015.

7. Mughal, A., C. Sun, and S.T. O'Rourke, Activation of Large Conductance, Calcium-Activated Potassium Channels by Nitric Oxide Mediates Apelin-Induced Relaxation of Isolated Rat

Coronary Arteries. J Pharmacol Exp Ther, 2018. 366(2): p. 265-273.

8. Tatin, F., et al., Apelin modulates pathological remodeling of lymphatic endothelium after myocardial infarction. JCl Insight, 2017. 2(12).

9. Romier, B., et al., Production of Elastin-Derived Peptides Contributes to the Development of Nonalcoholic Steatohepatitis. Diabetes, 2018. 67(8): p. 1604-1615.

10. Wahart, A., et al., Role of elastin peptides and elastin receptor complex in metabolic and cardiovascular diseases. FEBS J, 2019. 286(15): p. 2980-2993.

11. Maurice, P., et al., Elastin fragmentation and atherosclerosis progression: the elastokine concept. Trends Cardiovasc Med, 2013. 23(6): p. 211-21.

12. Duca, L., et al., Matrix ageing and vascular impacts: focus on elastin fragmentation.

Cardiovasc Res, 2016. 110(3): p. 298-308.

13. Fhayli, W., et al., Rise and fall of elastic fibers from development to aging. Consequences on arterial structure-function and therapeutical perspectives. Matrix Biol, 2019. 84: p. 41-56.

14. Trachet, B., et al., Ascending Aortic Aneurysm in Angiotensin II-Infused Mice: Formation, Progression, and the Role of Focal Dissections. Arterioscler Thromb Vasc Biol, 2016. 36(4): p.

673-81.

15. Roberts, D.L., C. Dive, and A.G. Renehan, Biological mechanisms linking obesity and cancer risk: new perspectives. Annu Rev Med, 2010. 61: p. 301-16.

16. Aboukais, R., et al., Ruptured blood blister like aneurysm: does the best therapeutic option really exist? Neurosurg Rev, 2021.

17. Wada, T., et al., Direct abdominal aortic access for thoracic endovascular aortic repair in a patient with severe aortic and arterial calcification. Ann Vasc Surg, 2020.

18. Queiroz, M. and C.M. Sena, Perivascular adipose tissue in age-related vascular disease. Ageing Res Rev, 2020. 59: p. 101040.

19. Liu, Y., et al., Perivascular Adipose Tissue as an Indication, Contributor to, and Therapeutic Target for Atherosclerosis. Front Physiol, 2020. 11: p. 615503.

20. Vink, R.G., et al., Adipose tissue gene expression is differentially regulated with different rates of weight loss in overweight and obese humans. Int J Obes (Lond), 2017. 41(2): p. 309-316. 
21. Lee, D.K., S.R. George, and B.F. O'Dowd, Unravelling the roles of the apelin system: prospective therapeutic applications in heart failure and obesity. Trends Pharmacol Sci, 2006. 27(4): p. 190-4.

22. Dalmas, E., et al., Intima-media thickness in severe obesity: links with BMI and metabolic status but not with systemic or adipose tissue inflammation. Diabetes Care, 2013. 36(11): p.

3793-802.

23. Naour, N., et al., Cathepsins in human obesity: changes in energy balance predominantly affect cathepsin s in adipose tissue and in circulation. J Clin Endocrinol Metab, 2010. 95(4): p.

1861-8.

24. Lafarge, J.C., et al., Cathepsins and cystatin C in atherosclerosis and obesity. Biochimie, 2010. 92(11): p. 1580-6.

25. Kozel, B.A. and R.P. Mecham, Elastic fiber ultrastructure and assembly. Matrix Biol, 2019. 84(2019): p. 31-40.

26. Chen, H., et al., Cathepsin S-mediated fibroblast trans-differentiation contributes to left ventricular remodelling after myocardial infarction. Cardiovasc Res, 2013. 100(1): p. 84-94.

27. Kehlet, S.N., et al., Cathepsin-S degraded decorin are elevated in fibrotic lung disorders development and biological validation of a new serum biomarker. BMC Pulm Med, 2017. 17(1): p. 110.

28. Yao, X., et al., Cathepsin $S$ regulates renal fibrosis in mouse models of mild and severe hydronephrosis. Mol Med Rep, 2019. 20(1): p. 141-150.

29. Blaise, S., et al., Elastin-derived peptides are new regulators of insulin resistance development in mice. Diabetes, 2013. 62(11): p. 3807-16.

30. Pooya, S., et al., Methyl donor deficiency impairs fatty acid oxidation through PGC-1alpha hypomethylation and decreased ER-alpha, ERR-alpha, and HNF-4alpha in the rat liver. J Hepatol, 2012. 57(2): p. 344-51.

\section{FIGURE LEGENDS}

Figure 1: Biochemical markers of adipogenicity and inflammatory in Apelin KO mice ( $\mathrm{n}=8$ /group): A, Body weight. B-C, Lean (B) and Fat (C) weights obtained by NMR-MRI-based technology. $\mathrm{D}$, mRNA expression of leptin and resistin in perigonadic white adipose tissue. E, mRNA expression of inflammatory markers including F4/80, MCP1 (monocyte chemoattractant protein 1), TNF $\alpha$ (Tumour Necrosis Factor), IL1 $\beta$ (interleukin) and TGF $\beta$ (transforming growth factor). G, mRNA expression of proteases such as neutrophil elastase (NE), Cathepsin S, MMP9 (metalloprotease). WT, white bars; KOAPL, grey bars. H, mRNA expression of protease inhibitors (serpin, cystatin C, TIMP1 (tissue inhibitors of metalloproteinase)). I, ratio between protease and its natural inhibitors. Results are the mean +/- SEM. Statistically significant differences (Mann-Whitney).

Figure 2: Apelin deficiency induce an arterial hypertension (n=8 /group): A, diastole and systole pressures measured by the tail cuff approach and performed one time per day for 4 days. WT, white bars; KO-APL, grey bars. B, mean arterial pressure (MAP) calculated from diastole and systole pressures. C, Pulse pressure correspond to the difference between systole and diastole pressures. D, Mean of pressures (systole and diastole) obtained on the 4 day of measures (ref to A panel). E, Mean of 
MAP obtained on the 4 day of measures (ref to B panel). F, Mean of pulse pression obtained on the 4 day of measures (ref to $\mathrm{C}$ panel). G, Heart Rate expressed in beat per minutes (BPM). H, Velocity of Propagation (VOP) of aorta measured by the pulse wave speed. I to K, Correlation between MCP1 mRNA expression issue to white adipose tissue (wat i.e. fig $1 \mathrm{~F}$ ) with systole (I, i.e. fig 2D) and pulse (J, i.e. fig $2 \mathrm{~F}$ ) pressures and VOP (K, i.e. fig $2 \mathrm{H}$ ). $\mathrm{L}$ to $\mathrm{N}$, Correlation between (Cathepsin S-Cystatin C) ratio issue to white adipose tissue (wat, i.e. fig 1I) with systole (L) and pulse (M) pressures and VOP (N). O to Q, Correlation between (Neutrophil Elastase (NE) - Serpin) ratio issue to white adipose tissue (wat, i.e. fig 1I) with systole $(\mathrm{O})$ and pulse $(\mathrm{P})$ pressures and VOP $(\mathrm{Q})$. Results are the mean +/- SEM. Statistically significant differences (Mann-Whitney).

Figure 3: Collagen expression in aorta of Apelin KO mice (n=8/group): A, Total collagen assay. B, mRNA expression of collagen I alpha. WT, white bars; KO-APL, grey bars. C, Representative of picrosirius staining observed by non-polarized light and obtained on 8 slices per mice and condition (panel a and b). Scale bars for panels a to d correspond to $250 \mu \mathrm{m}$. Panel $\mathrm{c}$ and $\mathrm{d}$ correspond to zoom of panel a and b. Scale bars correspond to $60 \mu \mathrm{m}$. D, Representative of picrosirius staining observed by polarized light (panel a and b). Scale bars correspond to $250 \mu \mathrm{m}$. Panel c and d correspond to zoom of panel a and b. Scale bars correspond to $60 \mu \mathrm{m}$. Scale bars for panels a to d correspond to $250 \mu \mathrm{m}$. Scale bars for panels e to h correspond to $60 \mu \mathrm{m}$. E, Semi-quantification of total collagen from picrosirius staining from panel C. F, Semi-quantification of collagen I and III from picrosirius staining from images. G, Correlation between MCP1 mRNA expression issue to white adipose tissue (wat, i.e. fig 1F) with collagen stained by picrosirius (i.e. fig $2 \mathrm{C}-\mathrm{E}$ ). $\mathrm{H}$ to J, Correlation between protease / inhibitor ratio determined in fig 1I with collagen stained by picrosirius (i.e. fig 2C-E). Results are the mean +/- SEM. Statistically significant differences (Mann-Whitney).

Figure 4: Spatial distribution of laminar ruptures in KO Apelin mice (n=8): A, Representative Hematoxin-Eosin (H\&E). Scale bar represents $250 \mu \mathrm{m}$. B, Scatter plots show the intima-media thickness and adventitia thickness. The mean and 95\% confidence interval are calculated in the normal domain after a $\log$ transformation. Three stains, each obtained by image-guided histology, were averaged per animal to account for intrasubject variation. $\mathrm{C}$, Representative autofluorescence of elastin (image of top panel). Scale bar represents $250 \mu \mathrm{m}$. Images of panel bottom correspond to a zoomed sections. Scale bar represents $60 \mu \mathrm{m}$. D, Bar plots indicate the number and thickness of lamina per aorta quadrant defined by Trachet al.[14]. Autofluorescence of elastin of full aorta section is quantified by Image-J. Three aorta section were averaged per animal to account for intrasubject variation. Laminar ruptures were calculated from 4 quadrants of 3 aorta sections per animals. E, Graph depicting linear relationships between measured elastin autofluorescence mean and mean of laminar rupture number obtained for each animal studied (WT, white dot and Ko, grey dot). F, Representation of numeration of elastic lamellae (L1 to L6, left panel). Lum, luminar. Number of laminar ruptures per quadrant (right 
panel). G, Plasma EDP assay (left), graphs depicting linear relationships 1- (middle panel) between measured plasma EDP and mean of laminar rupture number obtained for each animal studied; 2between plasma EDP and measured elastin autofluorescence mean obtained for each animal studied (WT, white dot and Ko, grey dot). H, Ratio calculated between measured elastin autofluorescence mean obtained for each animal and picrosirius staining obtained in figure 2. I, Univariate correlation obtained by Z-test between the ratio Elastin/Collagen obtained in figure $4 \mathrm{H}$ and the ratio Protease/its inhibitor determined in figure 1I. J, mRNA expression of Elastin, Fibrillin-1, Fibulin-5, obtained by real-time qPCR. Results are the mean +/- SEM. Statistically significant differences (Mann-Whitney).

Figure 5: Apelin deficiency increase the activity of Cathepsin $S$ in wall of aorta (n=8/group): A, Hematoxylin-Eosin staining and autofluorescence of elastin show a dissection in which the inner laminae (L1-L4) were affected, synonym to aneurism development. B, mRNA expressions of matrix metalloproteinase (MMP) -9 and 12, Neutrophil Elastase (NE), Cathepsin S (Cath. S). C, Bar plots indicate neutrophil elastase activity (left) and cathepsin S activity (right). D, Graph depicting linear relationships between measured neutrophil elastase activity and plasma EDP level, one the hand (right) and other the hand, mean of laminar rupture number obtained for each animal studied (right, WT, white dot and KO, grey dot). E, Graph depicting linear relationships between measured cathepsin S activity (left) and plasma EDP level, one the hand and other the hand, mean of laminar rupture number obtained for each animal studied (right, WT, white dot and KO, grey dot). F, Immunostanning against cathepsin $\mathrm{S}$ showing an accumulation in luminar $(\mathrm{L})$ near tunica intima and also between tunica media and tunica adventice (arrows). G, graph depicting linear relationships between measured cathepsin S staining and laminar rupture number (left) and elastin autofluorescence (right, WT, white dot and KO, grey dot). H, Immunostanning against TGF $\beta$ showing an accumulation in media in KO apelin mice. I, Graph depicting linear relationships between measured TGF $\beta$ staining and laminar rupture number (left) and elastin autofluorescence (right, WT, white dot and KO, grey dot). Results are the mean +/- SEM. Statistically significant differences (Mann-Whitney). 


\section{Figures}

A

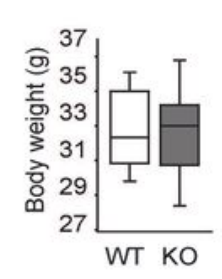

B

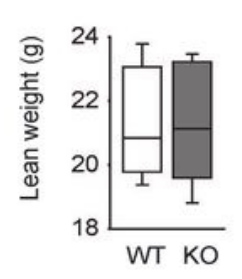

C
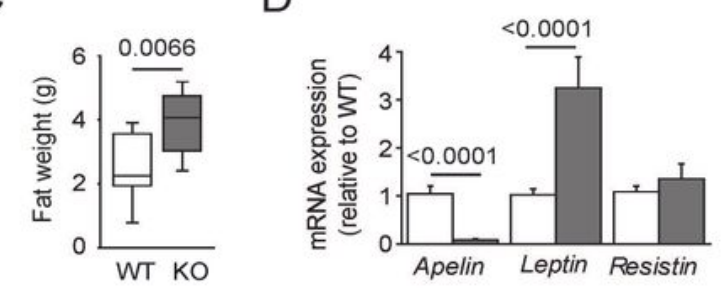

E

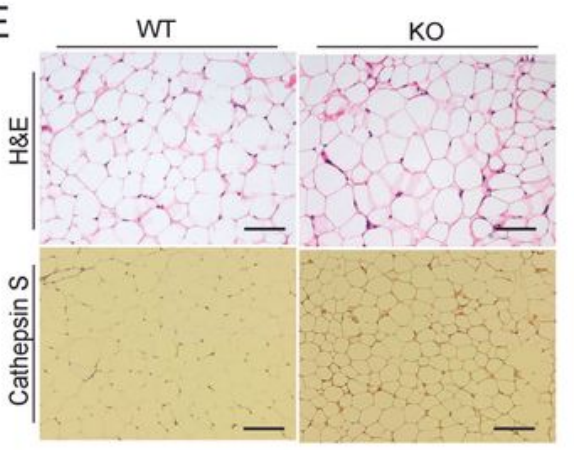

G

$\mathrm{H}$
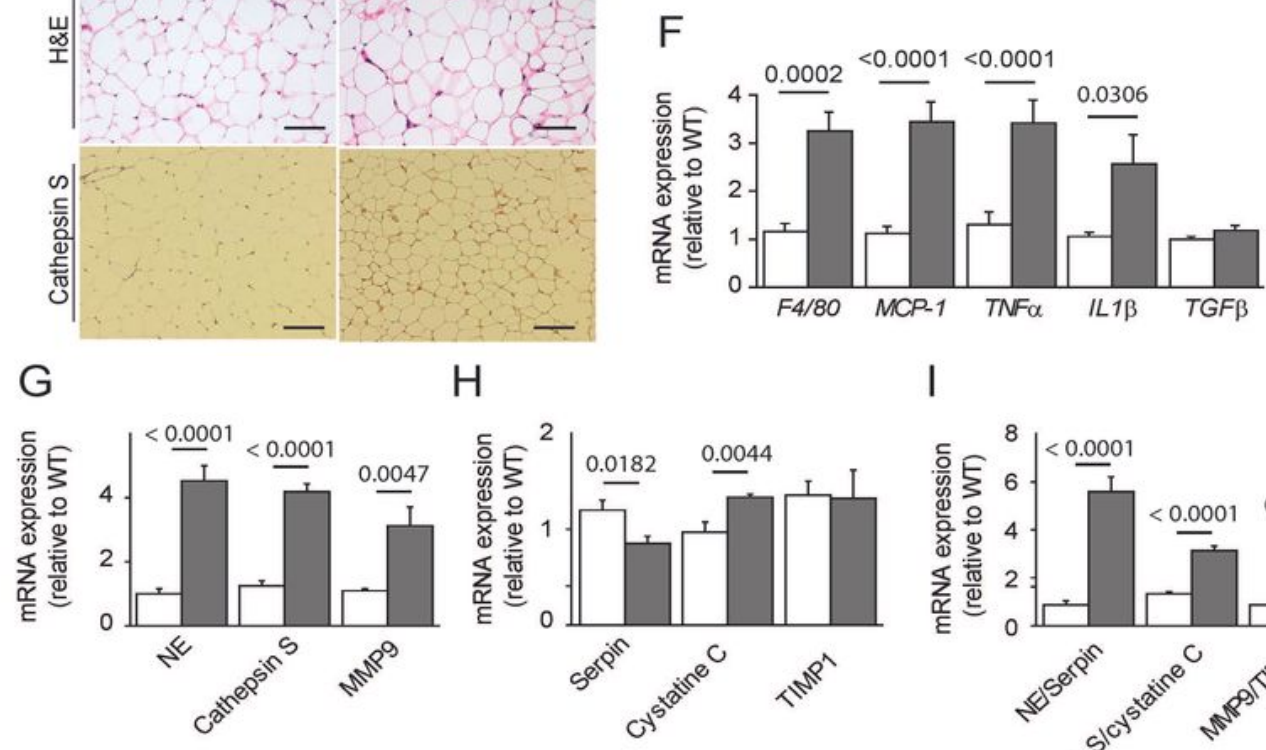

I

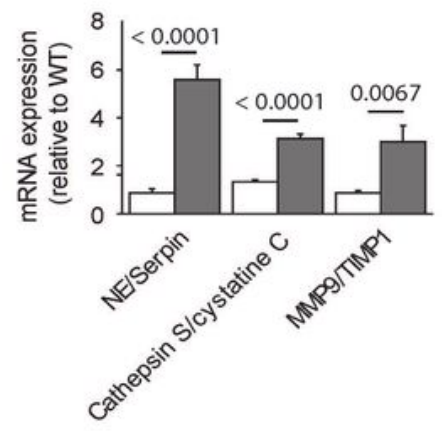

\section{FIGURE 1}

\section{Figure 1}

Biochemical markers of adipogenicity and inflammatory in Apelin KO mice ( $n=8$ /group): A, Body weight. B-C, Lean (B) and Fat (C) weights obtained by NMR-MRI-based technology. D, mRNA expression of leptin and resistin in perigonadic white adipose tissue. E, mRNA expression of inflammatory markers including 
F4/80, MCP1 (monocyte chemoattractant protein 1), TNFa (Tumour Necrosis Factor), IL1 $\beta$ (interleukin) and TGF $\beta$ (transforming growth factor). G, mRNA expression of proteases such as neutrophil elastase (NE), Cathepsin S, MMP9 (metalloprotease). WT, white bars; KO-APL, grey bars. H, mRNA expression of protease inhibitors (serpin, cystatin C, TIMP1 (tissue inhibitors of metalloproteinase)). I, ratio between protease and its natural inhibitors. Results are the mean +/- SEM. Statistically significant differences (Mann-Whitney).
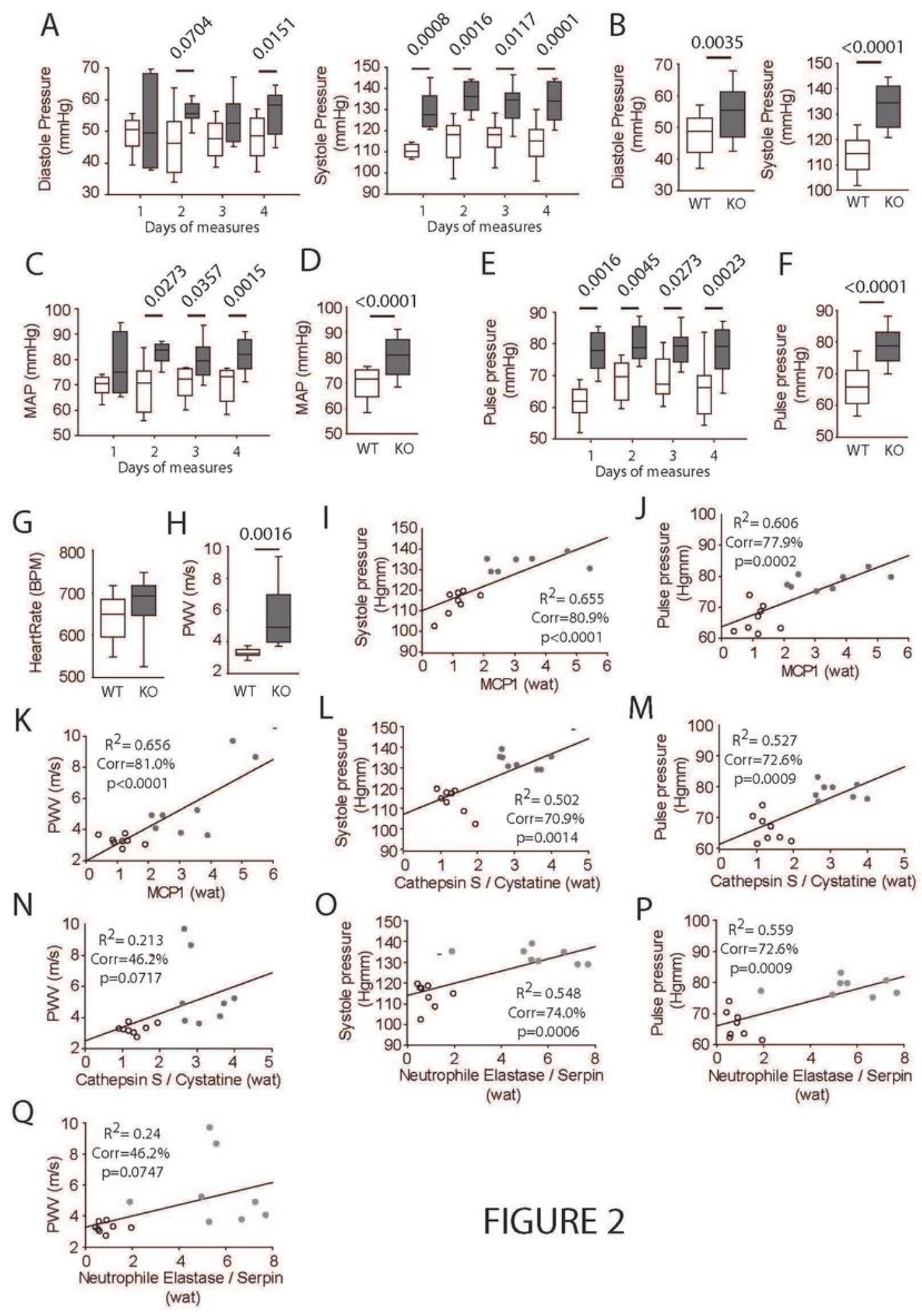

\section{FIGURE 2}

Figure 2 
Apelin deficiency induce an arterial hypertension ( $\mathrm{n}=8$ /group): A, diastole and systole pressures measured by the tail cuff approach and performed one time per day for 4 days. WT, white bars; KO-APL, grey bars. B, mean arterial pressure (MAP) calculated from diastole and systole pressures. C, Pulse pressure correspond to the difference between systole and diastole pressures. D, Mean of pressures (systole and diastole) obtained on the 4 day of measures (ref to A panel). E, Mean of MAP obtained on the 4 day of measures (ref to $B$ panel). F, Mean of pulse pression obtained on the 4 day of measures (ref to $C$ panel). G, Heart Rate expressed in beat per minutes (BPM). H, Velocity of Propagation (VOP) of aorta measured by the pulse wave speed. I to K, Correlation between MCP1 mRNA expression issue to white adipose tissue (wat i.e. fig $1 \mathrm{~F}$ ) with systole (I, i.e. fig 2D) and pulse (J, i.e. fig 2F) pressures and VOP (K, i.e. fig $2 \mathrm{H}$ ). $\mathrm{L}$ to $\mathrm{N}$, Correlation between (Cathepsin S-Cystatin $\mathrm{C}$ ) ratio issue to white adipose tissue (wat, i.e. fig 1I) with systole (L) and pulse (M) pressures and VOP (N). O to Q, Correlation between (Neutrophil Elastase (NE) - Serpin) ratio issue to white adipose tissue (wat, i.e. fig 1I) with systole (O) and pulse (P) pressures and VOP (Q). Results are the mean +/- SEM. Statistically significant differences (MannWhitney). 
A

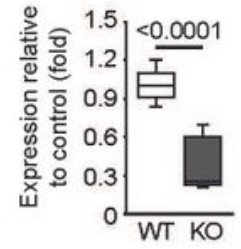

E

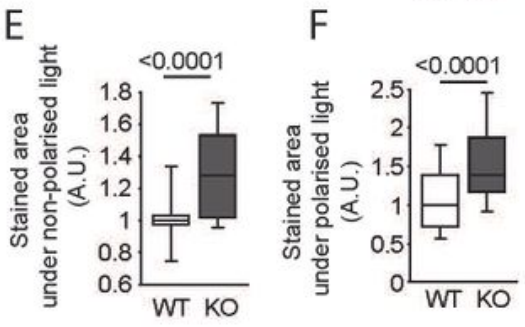

G

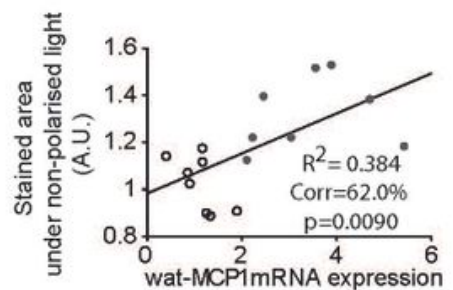

C

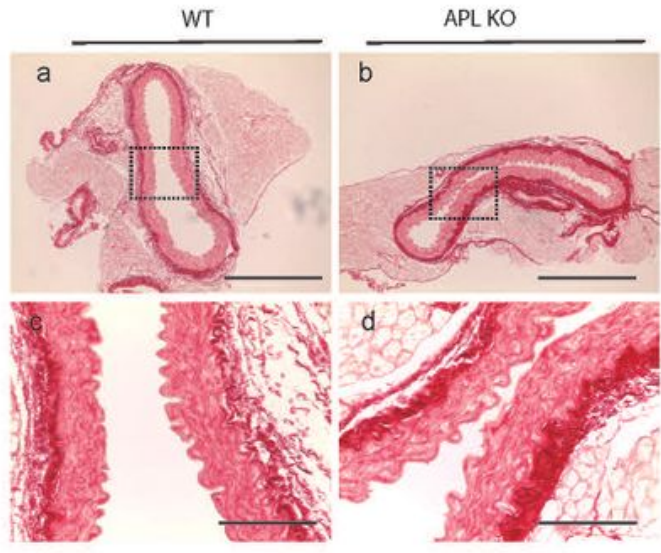

D
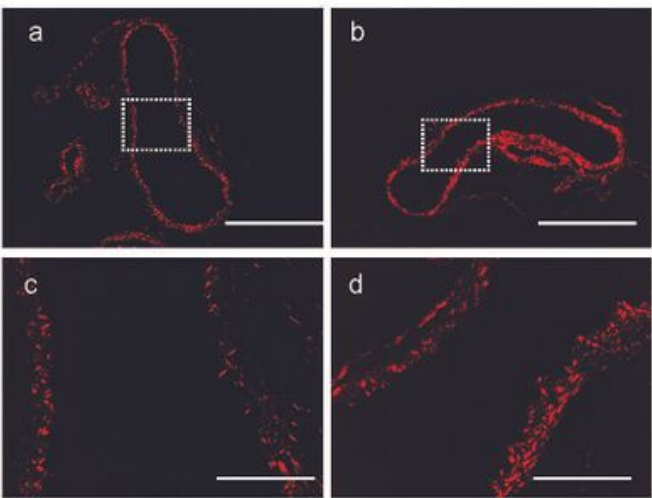

J

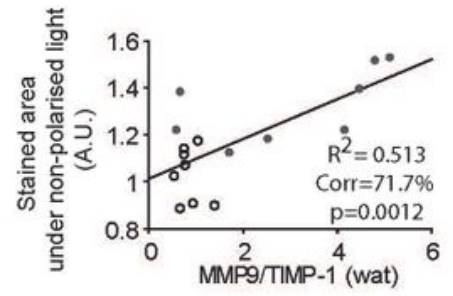

Figure 3

\section{Figure 3}

Collagen expression in aorta of Apelin KO mice ( $n=8 /$ group): $A$, Total collagen assay. $B$, mRNA expression of collagen I alpha. WT, white bars; KO-APL, grey bars. C, Representative of picrosirius staining observed by non-polarized light and obtained on 8 slices per mice and condition (panel a and b). Scale bars for panels a to $\mathrm{d}$ correspond to $250 \mu \mathrm{m}$. Panel $\mathrm{c}$ and d correspond to zoom of panel a and b. Scale bars correspond to $60 \mu \mathrm{m}$. D, Representative of picrosirius staining observed by polarized light (panel a and b). 
Scale bars correspond to $250 \mu \mathrm{m}$. Panel c and d correspond to zoom of panel a and b. Scale bars correspond to $60 \mu \mathrm{m}$. Scale bars for panels a to d correspond to $250 \mu \mathrm{m}$. Scale bars for panels e to $\mathrm{h}$ correspond to $60 \mu \mathrm{m}$. E, Semi-quantification of total collagen from picrosirius staining from panel C. F, Semi-quantification of collagen I and III from picrosirius staining from images. G, Correlation between MCP1 mRNA expression issue to white adipose tissue (wat, i.e. fig 1F) with collagen stained by picrosirius (i.e. fig $2 \mathrm{C}-\mathrm{E}$ ). $\mathrm{H}$ to $\mathrm{J}$, Correlation between protease / inhibitor ratio determined in fig $1 \mathrm{l}$ with collagen stained by picrosirius (i.e. fig 2C-E). Results are the mean +/- SEM. Statistically significant differences (Mann-Whitney). 

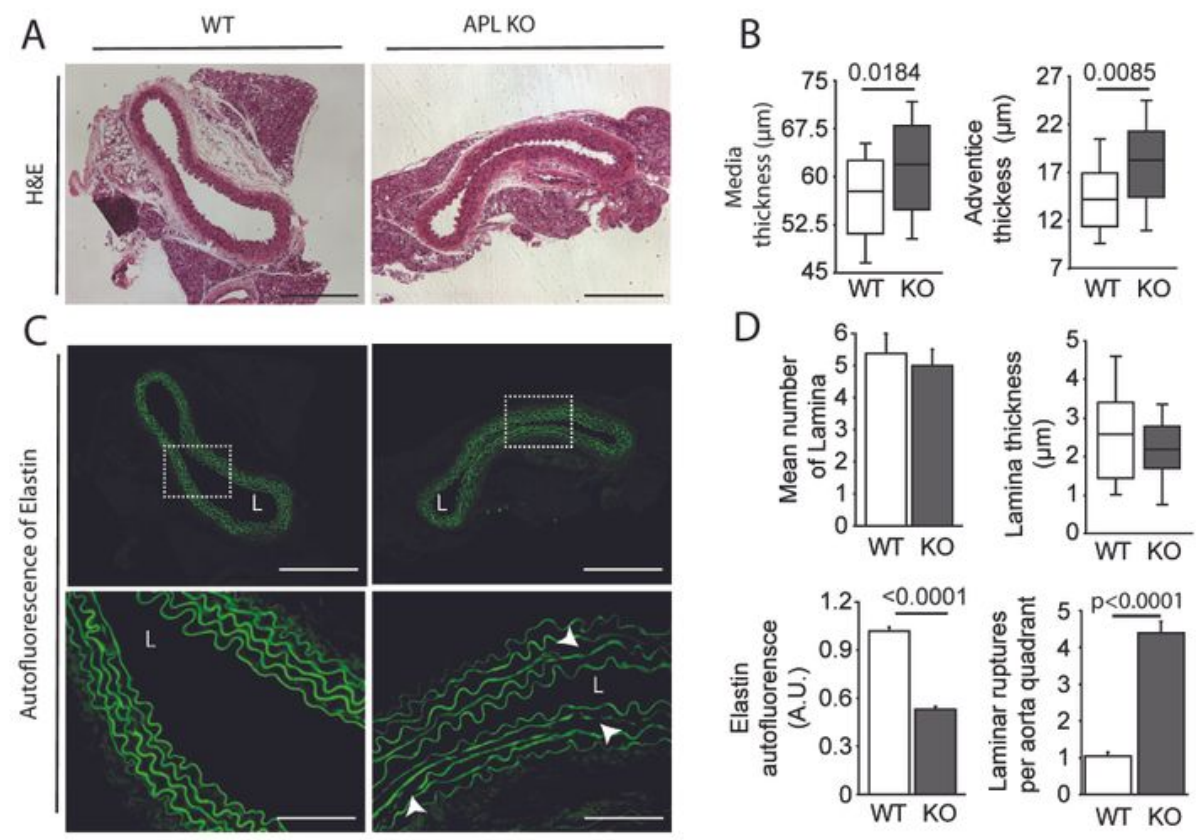

D
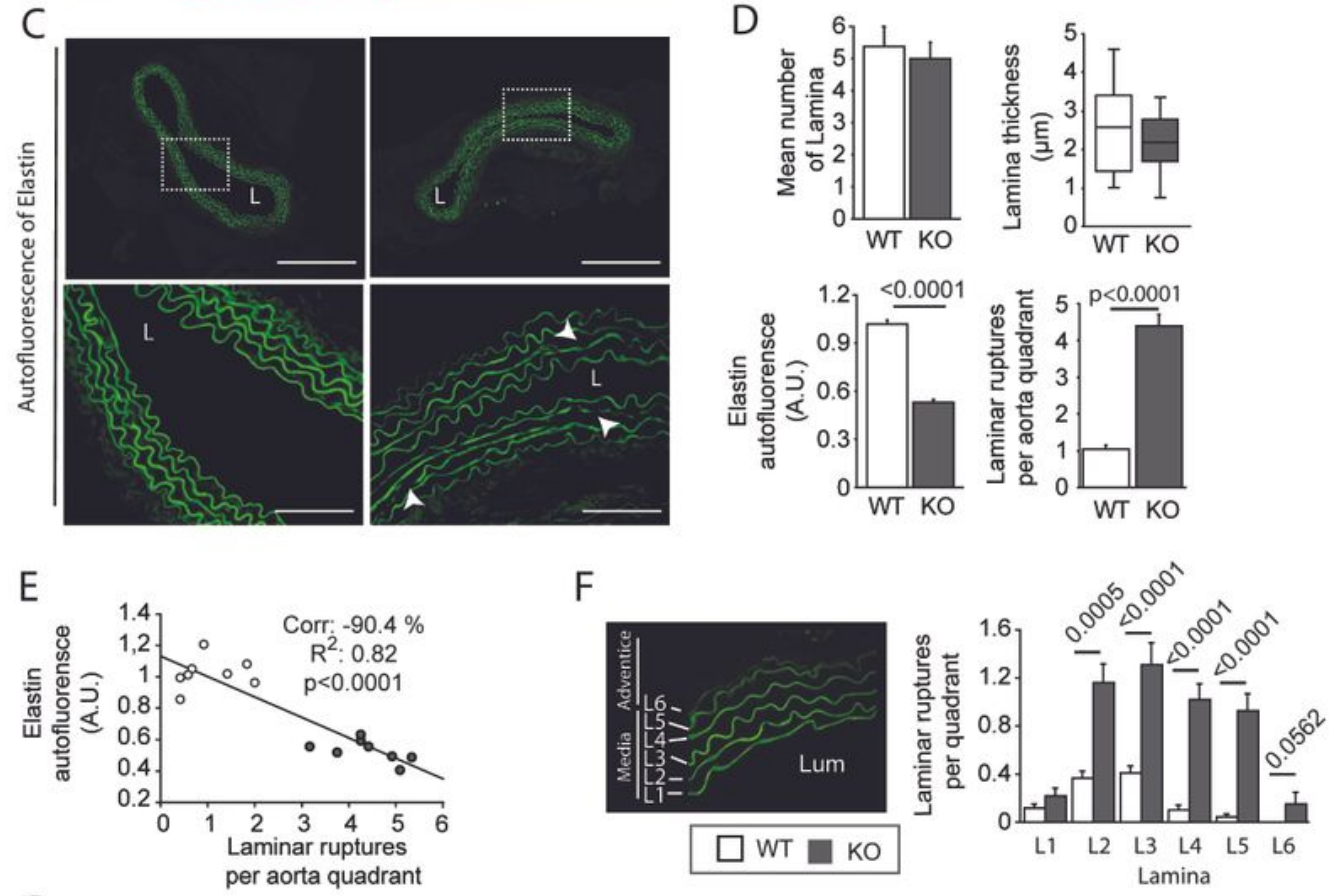

G
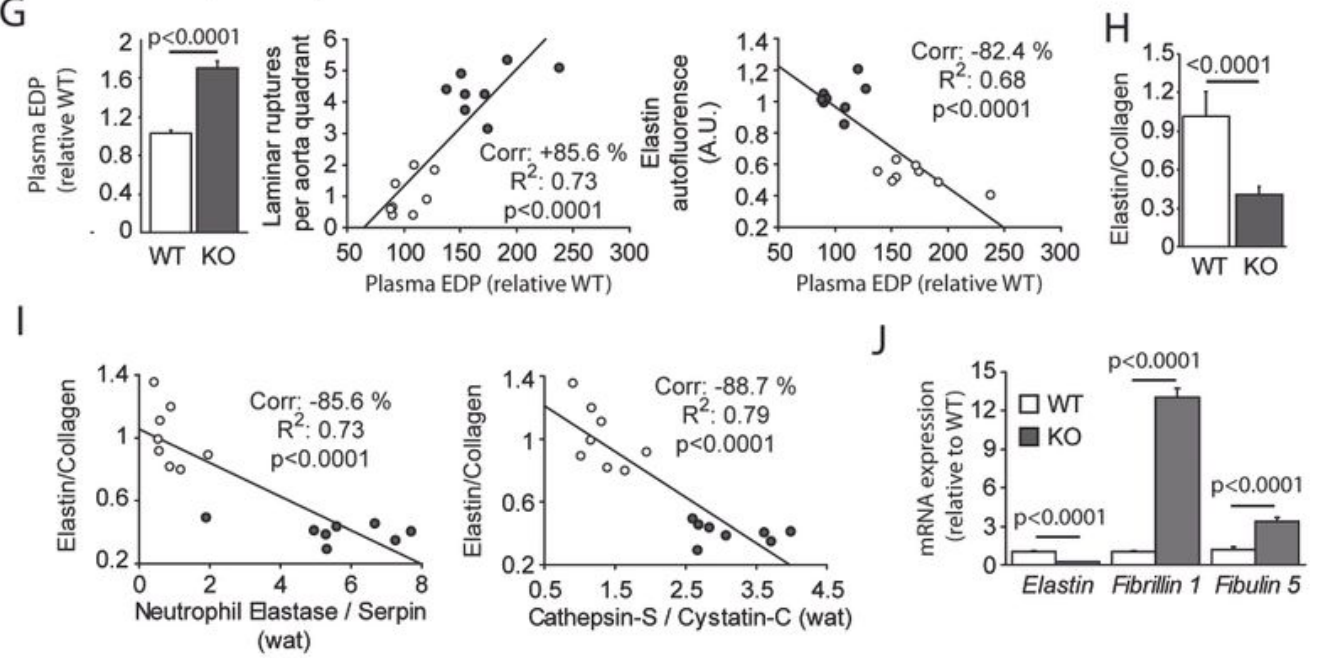

Figure 4

\section{Figure 4}

Spatial distribution of laminar ruptures in KO Apelin mice $(n=8)$ : A, Representative Hematoxin-Eosin (H\&E). Scale bar represents $250 \mu \mathrm{m}$. B, Scatter plots show the intima-media thickness and adventitia thickness. The mean and 95\% confidence interval are calculated in the normal domain after a log transformation. Three stains, each obtained by image-guided histology, were averaged per animal to account for intrasubject variation. C, Representative autofluorescence of elastin (image of top panel). 
Scale bar represents $250 \mu \mathrm{m}$. Images of panel bottom correspond to a zoomed sections. Scale bar represents $60 \mu \mathrm{m}$. D, Bar plots indicate the number and thickness of lamina per aorta quadrant defined by Trachet al.[14]. Autofluorescence of elastin of full aorta section is quantified by Image-J. Three aorta section were averaged per animal to account for intrasubject variation. Laminar ruptures were calculated from 4 quadrants of 3 aorta sections per animals. E, Graph depicting linear relationships between measured elastin autofluorescence mean and mean of laminar rupture number obtained for each animal studied (WT, white dot and Ko, grey dot). F, Representation of numeration of elastic lamellae (L1 to L6, left panel). Lum, luminar. Number of laminar ruptures per quadrant (right panel). G, Plasma EDP assay (left), graphs depicting linear relationships 1- (middle panel) between measured plasma EDP and mean of laminar rupture number obtained for each animal studied; 2- between plasma EDP and measured elastin autofluorescence mean obtained for each animal studied (WT, white dot and Ko, grey dot). H, Ratio calculated between measured elastin autofluorescence mean obtained for each animal and picrosirius staining obtained in figure 2. I, Univariate correlation obtained by Z-test between the ratio Elastin/Collagen obtained in figure $4 \mathrm{H}$ and the ratio Protease/its inhibitor determined in figure $1 \mathrm{l} . \mathrm{J}$, mRNA expression of Elastin, Fibrillin-1, Fibulin-5, obtained by real-time qPCR. Results are the mean +/SEM. Statistically significant differences (Mann-Whitney). 

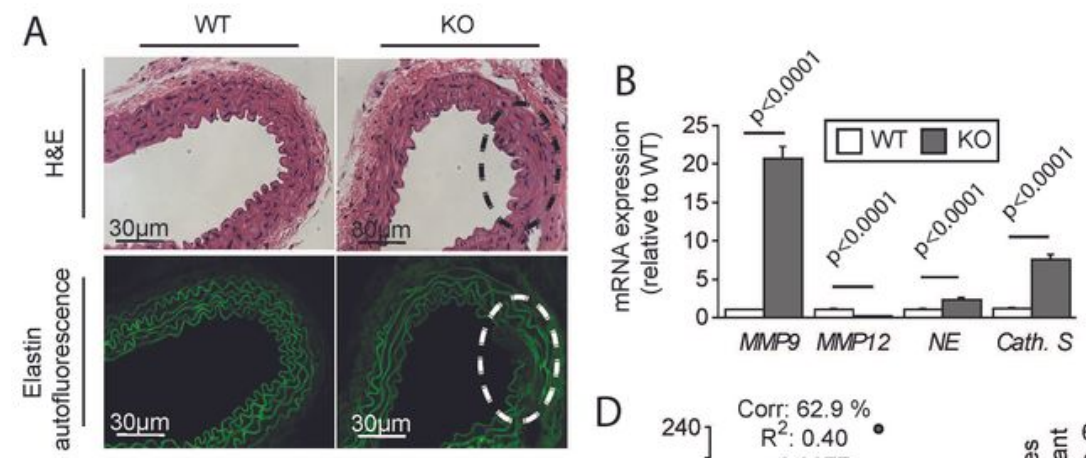

FIGURE 5

C
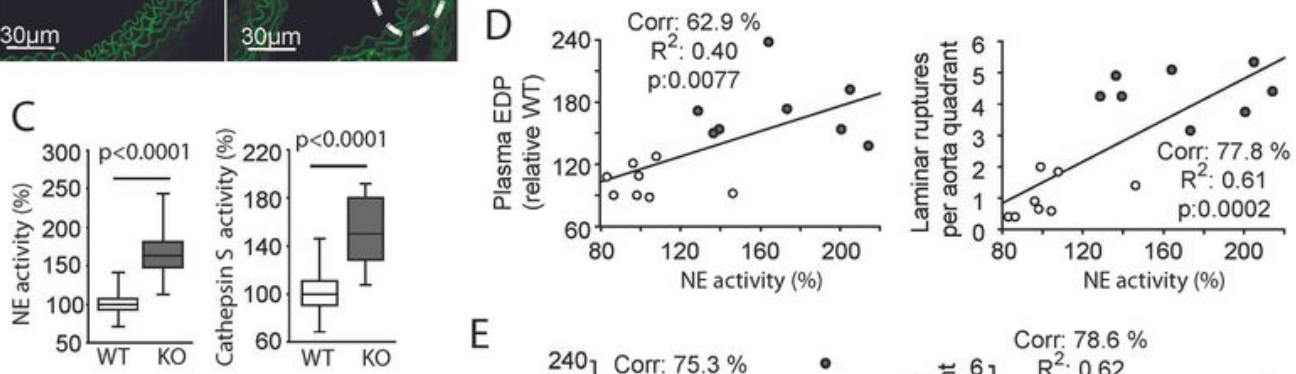

$\mathrm{E}$
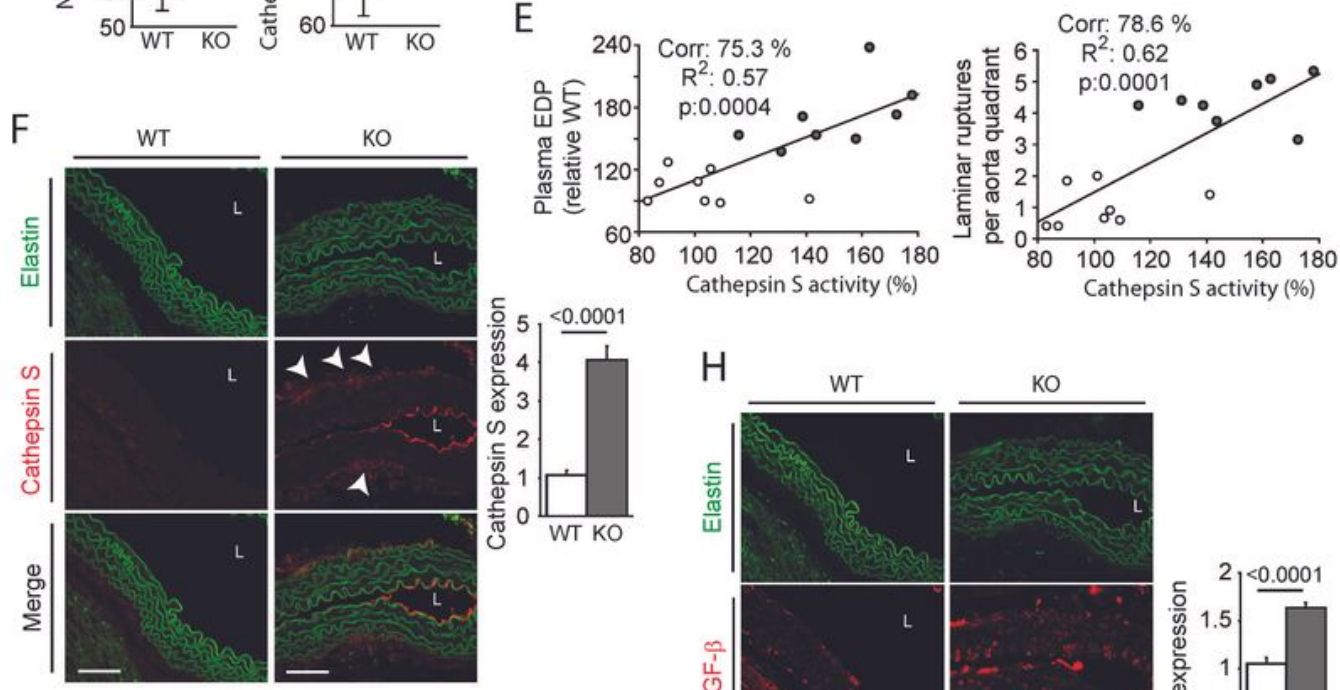

$\mathrm{H}$
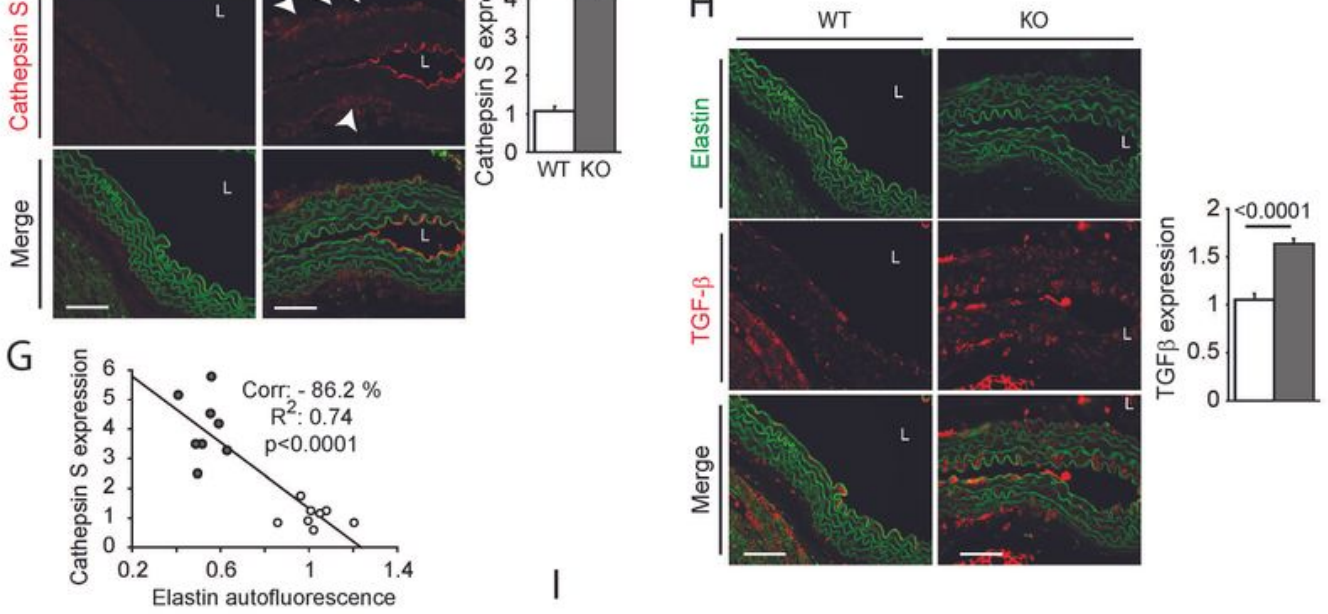

I
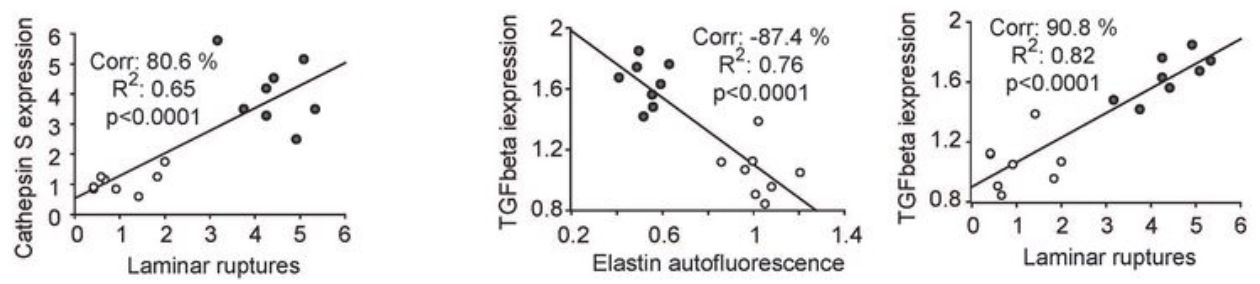

\section{Figure 5}

Apelin deficiency increase the activity of Cathepsin S in wall of aorta ( $n=8 /$ group): A, Hematoxylin-Eosin staining and autofluorescence of elastin show a dissection in which the inner laminae (L1-L4) were affected, synonym to aneurism development. B, mRNA expressions of matrix metalloproteinase (MMP) -9 and 12, Neutrophil Elastase (NE), Cathepsin S (Cath. S). C, Bar plots indicate neutrophil elastase activity (left) and cathepsin S activity (right). D, Graph depicting linear relationships between measured neutrophil 
elastase activity and plasma EDP level, one the hand (right) and other the hand, mean of laminar rupture number obtained for each animal studied (right, WT, white dot and KO, grey dot). E, Graph depicting linear relationships between measured cathepsin S activity (left) and plasma EDP level, one the hand and other the hand, mean of laminar rupture number obtained for each animal studied (right, WT, white dot and KO, grey dot). F, Immunostanning against cathepsin S showing an accumulation in luminar (L) near tunica intima and also between tunica media and tunica adventice (arrows). G, graph depicting linear relationships between measured cathepsin $\mathrm{S}$ staining and laminar rupture number (left) and elastin autofluorescence (right, WT, white dot and KO, grey dot). $\mathrm{H}$, Immunostanning against TGF $\beta$ showing an accumulation in media in KO apelin mice. I, Graph depicting linear relationships between measured TGF $\beta$ staining and laminar rupture number (left) and elastin autofluorescence (right, WT, white dot and KO, grey dot). Results are the mean +/-SEM. Statistically significant differences (Mann-Whitney).

\section{Supplementary Files}

This is a list of supplementary files associated with this preprint. Click to download.

- Table1envoi.pdf

- Suppldata1revu.pdf 